npj|Schizophrenia

\title{
ERRATUM
}

\section{The antiepileptic drug levetiracetam improves auditory gating in $\mathrm{DBA} / 2$ mice}

Jason Smucny, Karen E Stevens and Jason R Tregellas

npj Schizophrenia (2015) 1, Article number: 15053; doi:10.1038/npjschz.2015.53; published online 9 December 2015

Correction to: npj Schizophrenia 1, 15002 (2015). doi: 10.1038/ npjschz.2015.2

We are submitting an erratum for our published article 'The antiepileptic drug levetiracetam improves auditory gating in DBA/2 mice'. In the article abstract, the phrase 'Low-dose LEV may improve P20-N40 gating in the DBA/2 mouse model of schizophrenia' appears twice. The abstract should read:

'....No other doses significantly affected gating. Low doses of LEV may improve P20-N40 gating in the DBA/2 mouse model of schizophrenia and warrant further investigation in the illness.' 